\title{
Radiobiological doses, tumor, and treatment features influence on local control, enucleation rates, and survival after epiescleral brachytherapy. A 20-year retrospective analysis from a single- institution: part I
}

\author{
David Miguel, MPhys',2, Jesús Maria de Frutos-Baraja, PhD 1,2, Francisco López-Lara, PhD ${ }^{1,2}$, María Antonia Saornil, PhD 1,2, \\ Ciro Garcia-Álvarez, PhD 1,2, Pilar Alonso, PhD 1,2, Patricia Diezhandino, PhD',2 \\ Intraocular Tumor Unit, Hospital Universitario de Valladolid, Valladolid, ${ }^{2}$ University of Valladolid, Valladolid, Spain
}

\begin{abstract}
Purpose: To assess influence of the radiobiological doses, tumor, and treatment features on local control, enucleation rates, overall and disease-specific survival rates after brachytherapy for posterior uveal melanoma.

Material and methods: Local control, enucleation, overall and disease-specific survival rates were evaluated on the base of 243 patients from 1996 through 2016, using plaques loaded with iodine sources. Clinical and radiotherapy data were extracted from a dedicated prospective database. Biologically effective dose (BED) was included in survival analysis using Kaplan-Meier and Cox regressions. The 3-, 5-, 10-, and 15-year relative survival rates were estimated, and univariate/multivariate regression models were constructed for predictive factors of each item. Hazard ratio (HR) and confidence interval at $95 \%(\mathrm{CI})$ were determined.

Results: The median follow-up was 73.9 months (range, 3-202 months). Cumulative probabilities of survival by Kaplan-Meier analysis at 3, 5, 10 and 15 years were respectively: 96\%, 94\%, 93\%, and 87\%, for local control; 93\%, 88\%, $81 \%$, and $73 \%$ for globe preservation; $98 \%, 93 \%, 84 \%$, and $73 \%$ for overall survival, and $98 \%, 96 \%, 92 \%$, and $87 \%$ for disease-specific survival. By multivariate analysis, we concluded variables as significant: for local control failure the longest basal diameter and the juxtapapillary location; for globe preservation failure - the longest basal dimension, the mushroom shape, the location in ciliary body, and the dose to the foveola; for disease-specific survival - the longest basal dimension. Some radiobiological doses were significant in univariate models but not in multivariate ones for the items studied.

Conclusions: The results show as predictive factors of local control, enucleation, and disease-specific survival rates those related with the features of the tumor, specifically the longest basal dimension. There is no clear relation between radiobiological doses or treatment parameters in patients after brachytherapy.
\end{abstract}

J Contemp Brachytherapy 2018; 10, 4: 337-346 DOI: https://doi.org/10.5114/jcb.2018.77849

Key words: brachytherapy, disease-specific survival, enucleation, local control, radiobiological doses, uveal melanoma.

\begin{abstract}
Purpose
Uveal melanoma is the most common primary eye cancer in adults, affecting from 2 to 8 individuals per million in a year in Europe [1]. Once the primary tumor has been diagnosed, complementary tests should be performed to rule out the extension of the disease remotely, mainly by means of blood tests of the liver enzymes and radiological tests such as abdominal ultrasound or a chest computed tomography (CT) scan. At that time, less than
\end{abstract}

$2 \%$ of patients have detectable metastases [2]. However, the 5-year mortality rate for tumors smaller than $10 \mathrm{~mm}$ in diameter at its base is approximately $15 \%$, while for those larger than $15 \mathrm{~mm}$, it increases to $53 \%$ [3,4]. After metastasis, approximately $80 \%$ of the patients die after 1 year and $92 \%$ after 2 years. Metastasis had been documented 40 years after the first diagnosis and treatment of the primary tumor. This is due to ability of the disease to produce subclinical metastasis that, for an unknown reason, remain quiescent $[5,6]$.

Address for correspondence: David Miguel, MD, Intraocular Tumors Unit Valladolid University Hospital, Received: 06.04.2018 University of Valladolid, Spain, C/Avda. Ramón y Cajal s/n, 47003, Valladolid, 47003 Valladolid, Spain, Accepted: 25.07.2018 phone: +34 609965860, « e-mail: david.miguel@outlook.com 
The management of the posterior uveal melanoma depends on tumor size and location, and includes focal transpupillary thermotherapy for small borderline melanoma; resection for anterior tumors; radiotherapy (plaque radiotherapy, external beam radiation therapy or charged particle irradiation) for small, medium, and large melanoma, and enucleation for large melanoma or those encircling the optic disc $[7,8]$. The Collaborative Ocular Melanoma Study (COMS) showed that treatment with plaques loaded with ${ }^{125}$ I achieved local tumor control and survival comparable to enucleation [9].

The main purposes of brachytherapy are tumor control, eye preservation, maintenance of vision, and quality of life. The American Brachytherapy Society (ABS) recommended prescription dose is 85 Gy to the apex of the tumor [10] and to encompass small retinal-free margin typically at 2-3 $\mathrm{mm} \mathrm{[11]} \mathrm{around} \mathrm{the} \mathrm{tumor} \mathrm{base} \mathrm{(to} \mathrm{avoid}$ geographic miss), using COMS dosimetry assumptions [12] and plaque construction techniques [10,11]. A dose rate of $0.60-1.05 \mathrm{~Gy} / \mathrm{h}$ delivering the total dose in 3 to 10 consecutive days is suggested [11]. It should be noted that a $2 \mathrm{~mm}$ around margin can increase the plaque diameter by $4 \mathrm{~mm}$.

Secondary enucleation for reasons such as treatment failure (progressive tumor growth or tumor recurrence) or complications following treatment (painful eye, glaucoma, scleral necrosis, etc.) was evaluated in the context of brachytherapy failure. Radiation-induced ocular injury was not uncommon, and necessitated enucleation in approximately $5 \%$ of patients [13]. Although enucleation for such indications would not increase the risk of distant metastases [14], it represents a failure to achieve the goal of globe conservation.

Dose alone fails to represent the effect on biological tissues if it is delivered in different daily fractions, at a various dose rate or radiation quality [15]. In 1989, the British Journal of Radiology published a review suggesting the term of biologically effective dose (BED) based on linear quadratic cell survival in radiobiology [16]. Dale quantitatively evaluated the BED of non-permanent brachytherapy implants as a function of duration of an implant, the radionuclide of treatment, and radiobiological parameters for the tumor and organs at risk (OAR) related with repair rates and radiosensibility [17]. Finally, Gagne and colleagues developed a DVH model based on BED [18] and predicted theoretical outcomes [19].

Clinical optimization of eye plaque brachytherapy is currently limited to tumor coverage, consensus prescription dosage, and dose calculations to ocular structures. Enhanced clinical optimization should evaluate BED delivered to the tumor volume and surrounding normal ocular structures. Unfortunately, BED is generally not used in analyses of clinical series of patients treated with ophthalmic plaques.

The aim of this study was to analyze local control, enucleation rates, and survival rates for patients treated with ${ }^{125} \mathrm{I}$ brachytherapy at our center and correlate the outcomes with recognized prognostic factors related to the tumor features, physical, and radiobiological doses to the tumor and OAR and treatment characteristics.

\section{Material and methods}

\section{Patients diagnosis, treatment, and workflow}

All patients were initially evaluated and diagnosed with uveal melanoma by an ophthalmologist with expertise in ocular oncology. The diagnosis of choroidal melanoma and dimensions of the lesion were confirmed with ophthalmoscopic and ultrasonographic findings.

Ophthalmologist and oncologist contoured the target according to ultrasonographic findings and the plaque size was chosen to sufficiently encompass the basal margin. At time of diagnosis, all patients were evaluated by liver ultrasonography, chest radiography, and blood tests. Brachytherapy was performed according to a standard protocol following of the American Brachytherapy Society (ABS) guidelines [10,11].

The workflow was articulated in five main issues [20]: 1. Multidisciplinary tumor board: case presentation and treatment choice; 2 . Treatment planning: plan calculation and pre-plan approval; 3 . Source preparation: applicator loading and sterilization; 4 . Surgery: plaque implantation, treatment; 5. Plaque removal. If the lesion was near the muscle insertion area, the muscle was temporarily removed.

\section{Data collection and patient follow-up}

Data were collected from an intraocular tumor-dedicated database, which is part of a prospective study in our center for the last 20 years. Patients treated with ${ }^{125} \mathrm{I}$ (ROPES [21] and COMS [22]) plaques for uveal melanoma from $1^{\text {st }}$ of January 1996 to $1^{\text {st }}$ of July 2016 at the Intraocular Tumor Unit at Valladolid University Hospital, Spain, were included in this study. Patients treated with brachytherapy for iris and those treated with transpupillary thermotherapy (TTT) prior to brachytherapy were excluded.

Regular follow-up was scheduled at 1, 3, 6 and 12 months, every 6 months from 1 to 5 years after therapy, and then annually thereafter if local control had been achieved. In practice, the number of follow-ups may be bigger during the first 5 years, mainly due to special monitoring of some of the patients and also, the follow-up times may vary due to hospital planning.

Patient data included demographics (age and gender) and tumor characteristics (size by COMS criteria, apical height, longest basal dimension, laterality, length, latitude, location of anterior tumor border, location of posterior border, tumor shape, and juxtapapillary localization).

All patients signed an informed consent form for treatment after being properly informed about possible side effects. The study protocol and data collection were approved by the institutional research committee. The whole process was in accordance with national data protection laws, and the ethical principles of the Helsinki Declaration were applied.

\section{Clinical target volume definition and dosimetry}

Tumor shape and clinical target volume (CTV) was defined by the radiation oncologist, taking into account 
the tumor thickness read on AB-scan sonography images and a safety margin extension of $2-3 \mathrm{~mm}$ for the tumor basal dimension [12]. Planning target volume (PTV) can be added by radiation oncologist in case of doubts in plaque localization or tumor delineation [23].

Dosimetry and three-dimensional reconstruction were performed by a computer system developed by Dr. Astrahan at the University of California (BEBIG Plaque Simulator, version 2.16) [24]. Seed Amersham model 6711 [25,26] and Bebig model I25.S16 [25,26] were used for ROPES and COMS plaques, respectively. Plaque arrangements could vary in source strengths and ring sizes.

Calculations were based on the reports of the American Association of Physicists in Medicine Task Group No. $43[25,26]$. The dosimetry was performed consistently throughout the period studied. Corrections were made to the protocol to take into account changes in the dosimetry collected in TG-43 U1 [26], but the rest of assumptions have remained invariant in time. Plaque heterogeneity correction functions were incorporated in the treatment planning. Global attenuation factor that considered the effect of the eye plaque seed carrier and dose collimation by the lip on the gold-alloy backing were also enabled. An independent check of treatment time by redundant calculation was performed.

Before treatment, the following information were determined: treatment duration, plaque size, number of seeds, total air kerma rate of the plaque, and distribution of seeds required to provide the prescribed dose to the PTV. We also collected the initial dose rates and doses to prescription point, eye center (EC) $(12 \mathrm{~mm}$ from plaque center), sclera ( $1 \mathrm{~mm}$ from plaque center), and critical structures within the eye: lens (center of plaque to center of the lens), optic disc, (center of plaque to center of optic disc), and foveola (center of plaque to center of foveola).

\section{Biologically effective dose calculations}

BED equation for temporary brachytherapy implants was established by Dale [17] and Dale \& Jones [27]. BED includes, among other factors, initial dose rate, radionuclide half-life, tissue type, and repopulation terms. We can find the full equation in the Appendix of Verhoeff et al. [28] Eq. (D2) from Dale and Jones corrected a mathematical typo.
It is assumed that the repopulation rate remains constant and it is not needed for late-responding tissue [29]. Decay constant and all of the radiobiological values from Gagne et al. [18] were maintained.

Although Dale's formula was developed after our study, we were able to evaluate the BED to each of the interest points knowing the treatment time, the radiobiological values of the tissue, and the total physical dose delivered.

\section{Statistics}

Prognostic factors for local tumor control, eye preservation, overall, and disease-specific survival were evaluated by Kaplan-Meier and univariate/multivariate proportional hazards analysis as a function of dose to different tissues and characteristics features of the tumor. If survival exceeds $50 \%$ at the longest time point, then median survival cannot be computed.

For each item, survival times were estimated with 95\% confidence intervals (CI) by Kaplan-Meier analysis survival [30] and reported at 3, 5, 10, 15 years of follow-up. Cox regression modelling was used for univariate and multivariate analyses [31]. The effect of individual clinical variables was analyzed by a series of univariate Cox proportional hazards regressions. Hazard ratio (HR) and 95\% CI for variables with p-value less than 0.1 in the univariate analysis were entered into the final multivariate model with fitted variables identified as significant predictors in the backward stepwise model.

Local control failure was defined as the progression of the height of the tumor in $25 \%$, the increase of some of its margins or the presence of extrascleral extension as in COMS study [32].

All variables were analyzed as discrete variables except age, apical height, longest basal dimension, activity and treatment time, plaque size, radiation doses and BEDs, which were analyzed as continuous variables. Data were summarized as mean, standard deviation (SD), median, and interquartile range (IQR) for continuous variables and proportions for categorical variables. Outliers data (dose to tumor apex below $70 \mathrm{~Gy}$ due to unfinished treatments) were removed.

To allow for multiple comparisons, the level of statistical significance was set at $p<0.05$. All analyses were conducted using SPSS version 24.0 (IBM, Somers, NY, USA) and XLSTAT version 2016.02.28451 (Addinsoft).

Table 1. Tumor and treatment features. Quantitative variables. $N=243$

\begin{tabular}{|c|c|c|c|c|c|c|}
\hline Variable & Minimum & Maximum & Mean & SD & Median & IQR \\
\hline Age (years) & 16.00 & 91.00 & 59.74 & 13.47 & 61.00 & $50.00-70.00$ \\
\hline Tumor apical height (mm) & 1.00 & 12.11 & 5.68 & 2.45 & 5.45 & $3.60-7.63$ \\
\hline Longest basal dimension (mm) & 5.00 & 20.47 & 11.50 & 2.75 & 11.72 & $9.40-13.71$ \\
\hline Total source strength $(U)$ & 13.56 & 136.25 & 59.66 & 24.02 & 44.68 & $40.55-76.00$ \\
\hline Treatment time (h) & 52.00 & 283.00 & 129.50 & 43.84 & 120.00 & $95.00-167.00$ \\
\hline Size of the plaque $(\mathrm{mm})$ & 11.00 & 22.00 & 16.01 & 2.27 & 16.00 & $14.00-18.00$ \\
\hline
\end{tabular}

$S D$ - standard deviation, IQR - interquartile range 
Table 2. Tumor and treatment features. Qualitative variables. $N=243$

\begin{tabular}{|c|c|c|}
\hline Variable/statistic & $n$ & $\%$ \\
\hline \multicolumn{3}{|l|}{ COMS } \\
\hline Large & 18 & 7.44 \\
\hline Medium & 225 & 92.59 \\
\hline \multicolumn{3}{|l|}{ Gender } \\
\hline Female & 127 & 52.26 \\
\hline Male & 116 & 47.74 \\
\hline \multicolumn{3}{|l|}{ Laterality } \\
\hline Right eye & 125 & 51.44 \\
\hline Left eye & 118 & 48.56 \\
\hline \multicolumn{3}{|l|}{ Length } \\
\hline Nasal & 60 & 24.69 \\
\hline Temporal & 183 & 75.31 \\
\hline \multicolumn{3}{|l|}{ Latitude } \\
\hline Inferior & 105 & 43.21 \\
\hline Superior & 138 & 56.79 \\
\hline \multicolumn{3}{|c|}{ Location of anterior tumor border } \\
\hline Ciliary body & 24 & 9.87 \\
\hline Equator to ora serrata & 97 & 39.92 \\
\hline Posterior to equator & 122 & 50.21 \\
\hline \multicolumn{3}{|l|}{ Location of posterior border } \\
\hline$<1 \mathrm{~mm}$ OD & 26 & 10.69 \\
\hline$>1 \mathrm{~mm} \mathrm{OD}$ & 207 & 85.18 \\
\hline Equator to ora serrata & 10 & 4.11 \\
\hline \multicolumn{3}{|l|}{ Tumor shape } \\
\hline Mushroom & 58 & 23.87 \\
\hline Diffuse & 2 & 0.82 \\
\hline Nodular & 183 & 75.31 \\
\hline \multicolumn{3}{|l|}{ Juxtapapillary localization* } \\
\hline No & 208 & 85.60 \\
\hline Yes & 35 & 14.40 \\
\hline \multicolumn{3}{|l|}{ Shape plate } \\
\hline Not notched & 209 & 86.01 \\
\hline Notched & 34 & 13.99 \\
\hline \multicolumn{3}{|l|}{ Type of plate } \\
\hline COMS & 183 & 75.31 \\
\hline ROPES & 60 & 24.69 \\
\hline
\end{tabular}

*uxtapapillary choroidal melanoma is considered with a posterior margin within $1 \mathrm{~mm}$ from the optic disc (OD)

\section{Results}

\section{Patients}

From 1996 through June 2016, 714 patients with iris, choroidal, or ciliary body melanomas were diagnosed and 303 were treated by brachytherapy. 20 patients $(6.6 \%)$ were not eligible for the study because a ruthenium plaque treatment was performed, $38(12.5 \%)$ patients were treated with TTT or were lost to follow-up, and $2(0.6 \%)$ finished the treatment prematurely due to complications. Ultimately, 243 patients have met the inclusion criteria. The median follow-up was 74.5 months (range, 3-202).

Table 1 and 2 display baseline patient demographic and tumor characteristics. Table 3 displays the doses to the tumor apex, macula, optic disc, sclera, and eye center for the study population.

\section{Local tumor control}

From our cohort of 243 patients, recurrence was known for 13 (5.3\%) patients. Actuarial Kaplan-Meier curves and confidence intervals are described in Figure 1. The 3-, 5-, 10-, and 15-year probability for no local recurrence were 96\% (95\% CI: 95-100\%), 94\% (95\% CI: 91-97\%), 93\% (95\% CI: 89-97\%), and 87\% (95\% CI: 77-97\%), respectively (Figure 1). Mortality is presented in Table 4.

Increased risk of local recurrence in univariate logistic regression analysis (Table 5) depends only on the juxtapapillary localization and the longest basal dimension. Multivariate logistic regression analysis, including age, showed that both, longest basal dimension (HR: 1.254, 95\% CI: 1.020-1.254, $p<0.032)$ and juxtapapillary localization (HR: 3.382, 95\% CI: 1.101-10.386, $p=0.033$ ) were significant (Table 6).

\section{Enucleation}

Information on eye preservation was known for 213 patients at the end of follow-up. Thirty patients eventually underwent enucleation because of local recurrence $(n=13 ; 5.3 \%$ of all eyes $/ 43 \%$ of all enucleations) and side effects ( $n=17 ; 7.0 \%$ of all eyes $/ 57 \%$ of all enucleations) despite local tumor control achieved. All 13 patients with local failure eventually required enucleation. The 3-, 5-, $10-$, and 15-year actuarial enucleation-free rate showed a probability of $93 \%$ (95\% CI: 90-96\%), 88\% (95\% CI: 84-92\%), 81\% (95\% CI: 74-88\%), and 73\% (95\% CI: 62-84\%), respectively (Figure 1). Mortality is shown in Table 4.

Univariate factors predictive of enucleation are listed in Table 5 showing that tumor and treatment features, doses and BEDs to the foveola, sclera, and eye center were statistically significant. Multivariate logistic regression analysis, including age, revealed that longest basal dimension (HR: 1.190, 95\% CI: 1.002-1.412, $p=0.047$ ), dose to foveola (HR: 1.010, 95\% CI: 1.005-1.015, $p<0.000$ ), mushroom tumor shape vs. nodular (HR: 3.159 , 95\% CI: 2.542-6.472, $p=0.002)$, and location of anterior tumor border in ciliary body vs. equator to ora serrata (HR: 2.932, 95\% CI: 1.030-8.344, $p=0.011$ ) were significant (Table 6). BEDs dependencies vanished when multivariate competing risks regression modeling was performed. 
Table 3. Dosimetric characteristics of plaque treatments of the cohort. $N=243$

\begin{tabular}{lcccccc} 
& Minimum & Maximum & Mean & SD & Median & IQR \\
\hline Dose to tumor apex (Gy) & 74.65 & 95.71 & 85.31 & 3.61 & 85.14 & $83.99-86.89$ \\
\hline BED to tumor apex (Gy) & 86.62 & 149.11 & 108.88 & 10.39 & 109.27 & $100.52-116.04$ \\
\hline Dose to optic nerve (Gy) & 2.93 & 228.70 & 36.30 & 27.31 & 31.36 & $17.40-47.5$ \\
\hline BED to optic nerve (Gy) & 3.05 & 1040.21 & 75.70 & 101.42 & 51.08 & $24.39-93.01$ \\
\hline Dose to lens (Gy) & 2.90 & 85.32 & 19.36 & 13.77 & 16.25 & $9.17-26.15$ \\
\hline BED to lens (Gy) & 3.21 & 221.23 & 33.39 & 32.41 & 22.51 & $12.08-41.33$ \\
\hline Dose to foveola (Gy) & 3.17 & 418.20 & 51.84 & 50.90 & 36.43 & $20.94-63.39$ \\
\hline BED to foveola (Gy) & 3.27 & 2171.31 & 123.11 & 212.54 & 52.53 & $27.50-126.60$ \\
\hline Dose to sclera (Gy) & 3.26 & 722.00 & 294.14 & 131.38 & 258.50 & $205.80-369.50$ \\
\hline BED to sclera (Gy) & 140.10 & 4575.32 & 1330.15 & 846.55 & 1144.69 & $762.91-1739.52$ \\
\hline Dose to eye center (Gy) & 7.74 & 85.62 & 31.52 & 15.68 & 28.59 & $19.37-41.85$
\end{tabular}

$B E D$ - biologically effective dose, SD - standard deviation, $I Q R$ - interquartile range

CSF: Local control achieved

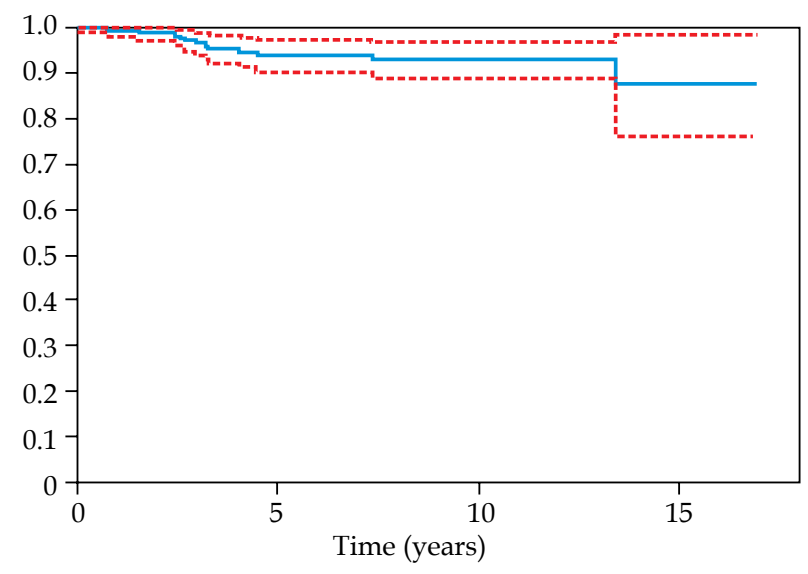

CSF: Global survival

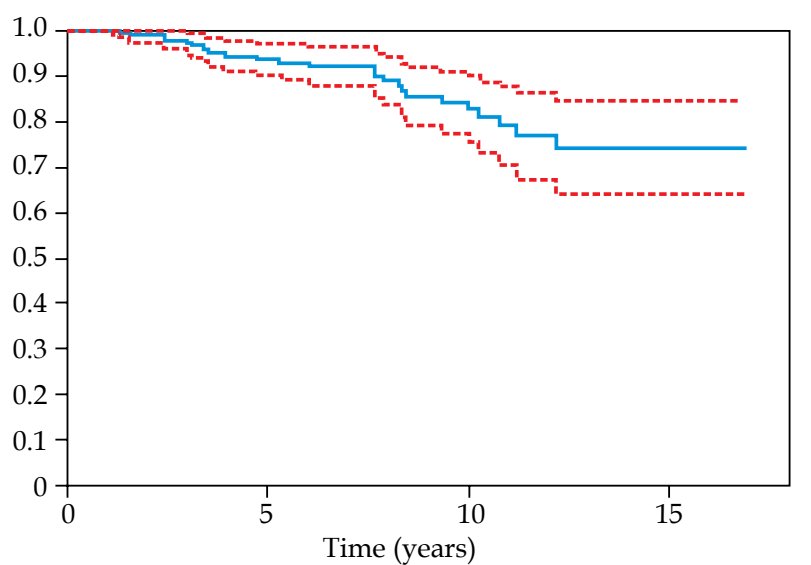

CSF: Enucleation-free survival

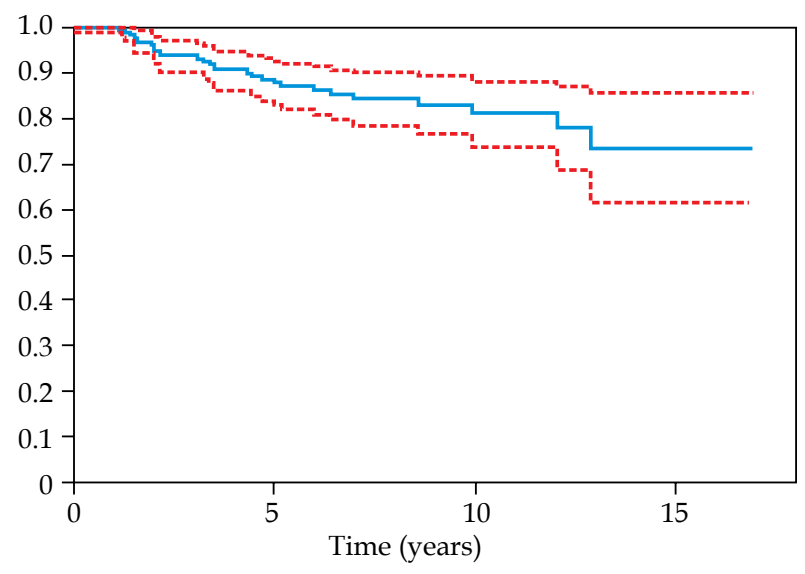

CSF: Disease-specific survival

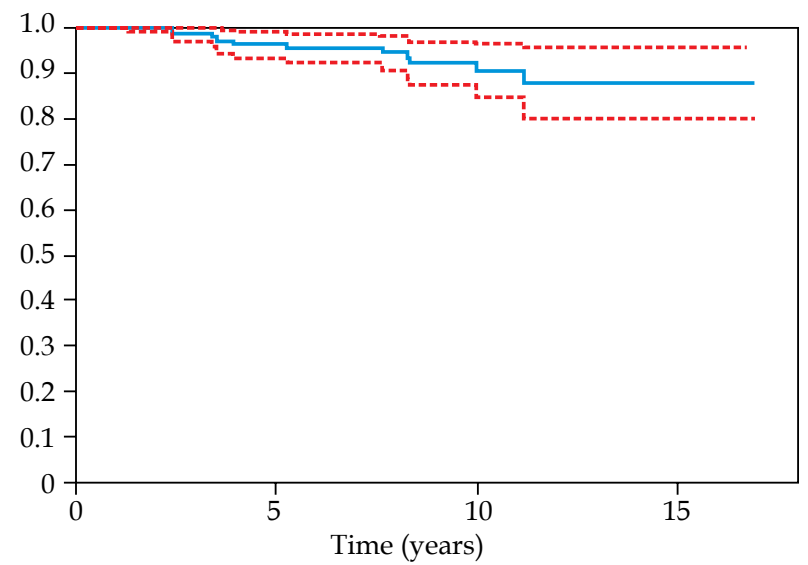

Fig. 1. Kaplan-Meier cumulative survival functions (CSF) for local control achieved, enucleation-free survival, global survival, and disease-specific survival (blue solid line) with confidence interval (red dashed line)

\section{Overall and disease-specific survival}

At the end of follow-up, the vital status was known for all patients, and a total of 26 patients $(10.4 \%)$ were dead. Thirteen deaths (46.4\% of all deaths) were melanoma-related due to systemic metastases.

The 3-, 5-, 10-, and 15-year melanoma overall survival rates were $98 \%$ (95\% CI: 97-99\%), 93\% (95\% CI: 90-96\%), 
Table 4. Mortality table for local control achieved, enucleation-free survival, global survival, and disease-specific survival. $T(y r)$ is the time of follow-up in years, $n$ is the number of patients at risk, and $S P$ is the survival probability (\%)

\begin{tabular}{|c|c|c|c|c|c|c|c|c|c|c|c|c|c|c|c|c|c|c|}
\hline Event & (yr) & 0 & 1 & 2 & 3 & 4 & 5 & 6 & 7 & 8 & 9 & 10 & 11 & 12 & 13 & 14 & 15 & 16 \\
\hline \multirow[t]{2}{*}{ Local control } & $n$ & 243 & 224 & 92 & 160 & 45 & 126 & 111 & 95 & 73 & 61 & 48 & 37 & 29 & D) & 2 & 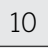 & 5 \\
\hline & SP & 100 & 99 & 99 & 96 & 95 & 94 & y & 94 & 9 & 93 & 93 & 9 & 93 & 93 & 87 & 7 & 87 \\
\hline \multirow{2}{*}{$\begin{array}{l}\text { Enucleation- } \\
\text { free survival }\end{array}$} & $n$ & 243 & 225 & 187 & 155 & 137 & 118 & 102 & 85 & 66 & 55 & 43 & 32 & 26 & 17 & 10 & 8 & 3 \\
\hline & SP & 100 & 100 & 96 & 93 & 90 & 88 & 87 & 85 & 8 & 82 & 81 & 8 & 8 & 7 & 73 & $7=$ & 73 \\
\hline \multirow[t]{2}{*}{ Overall survival } & $n$ & 243 & 229 & 195 & 169 & 151 & 132 & 117 & 102 & 81 & 67 & 52 & 40 & 31 & $\angle 2$ & 13 & 11 & 6 \\
\hline & SP & 100 & 100 & 99 & 98 & 94 & 93 & 93 & 92 & 89 & 8 & 84 & 78 & 76 & 73 & 73 & 73 & 73 \\
\hline \multirow{2}{*}{$\begin{array}{l}\text { Disease-specific } \\
\text { survival }\end{array}$} & $\pi$ & 243 & 229 & S & 169 & 151 & 132 & 117 & 102 & 81 & 67 & 52 & 4 & 31 & 22 & 10 & 11 & 6 \\
\hline & SP & 100 & 100 & 100 & 98 & 96 & 96 & 95 & 95 & 94 & 92 & 92 & 90 & 87 & 87 & 87 & 87 & 87 \\
\hline
\end{tabular}

Table 5. Univariable Cox regression analysis of visual local control, enucleation, and disease-specific survival. Variables with $p$-value less than 0.1 in the univariate analysis were shown

\begin{tabular}{|c|c|c|c|c|}
\hline \multirow[t]{2}{*}{ Factor } & \multirow[t]{2}{*}{$p$} & \multirow[t]{2}{*}{$\mathrm{HR}$} & \multicolumn{2}{|c|}{$95 \% \mathrm{Cl}$ for $\mathrm{HR}$} \\
\hline & & & Lower & Top \\
\hline \multicolumn{5}{|l|}{ Local control } \\
\hline Juxtapapillary localization (yes vs. no) & 0.050 & 3.054 & 0.998 & 9.344 \\
\hline Longest basal dimension (mm) & 0.062 & 1.228 & 0.990 & 1.522 \\
\hline \multicolumn{5}{|l|}{ Enucleation } \\
\hline Tumor apical height (mm) & 0.005 & 1.241 & 1.068 & 1.443 \\
\hline Longest basal dimension (mm) & 0.008 & 1.210 & 1.050 & 1.395 \\
\hline Total source strength $(U)$ & 0.031 & 1.296 & 1.272 & 1.321 \\
\hline Dose to foveola (Gy) & 0.002 & 1.007 & 1.003 & 1.012 \\
\hline BED to foveola (Gy) & 0.001 & 1.002 & 1.001 & 1.003 \\
\hline Dose to eye center (Gy) & 0.031 & 1.024 & 1.002 & 1.047 \\
\hline Dose to sclera (Gy) & 0.005 & 1.004 & 1.001 & 1.006 \\
\hline BED to sclera (Gy) & 0.002 & 1.001 & 1.000 & 1.001 \\
\hline \multicolumn{5}{|l|}{ Tumor shape } \\
\hline Mushroom (ref) & 0.007 & & & \\
\hline Nodular & 0.002 & 3.159 & 1.542 & 6.472 \\
\hline Diffuse & 0.984 & 0.000 & 0.000 & 0.000 \\
\hline \multicolumn{5}{|l|}{ Location of anterior tumor border } \\
\hline Ciliary body (ref) & 0.131 & & & \\
\hline Equator to ora serrata & 0.044 & 2.932 & 1.030 & 8.344 \\
\hline Posterior to equator & 0.419 & 1.382 & 0.630 & 3.031 \\
\hline \multicolumn{5}{|l|}{ Disease-specific survival } \\
\hline Longest basal dimension (mm) & 0.040 & 1.250 & 1.011 & 1.547 \\
\hline Total source strength $(\mathrm{U})$ & 0.005 & 1.042 & 1.013 & 1.073 \\
\hline Dose to lens (Gy) & 0.030 & 1.034 & 1.003 & 1.066 \\
\hline BED to lens (Gy) & 0.030 & 1.013 & 1.001 & 1.024 \\
\hline Dose to eye center (Gy) & 0.034 & 1.036 & 1.003 & 1.071 \\
\hline Size of plaque (mm) & 0.003 & 1.626 & 1.176 & 2.248 \\
\hline
\end{tabular}


Table 6. Multivariate model fitted variables identified as significant predictors in the backward stepwise model for local control, enucleation, and disease-free survival

\begin{tabular}{|c|c|c|c|c|}
\hline \multirow[t]{2}{*}{ Factor } & \multirow[t]{2}{*}{$p$} & \multirow[t]{2}{*}{$\mathrm{HR}$} & \multicolumn{2}{|c|}{$95 \% \mathrm{Cl}$ for $\mathrm{HR}$} \\
\hline & & & Lower & Top \\
\hline \multicolumn{5}{|l|}{ Local control } \\
\hline Juxtapapillary localization (yes vs. no) & 0.033 & 3.382 & 1.101 & 10.386 \\
\hline Longest basal dimension (mm) & 0.032 & 1.254 & 1.020 & 1.542 \\
\hline \multicolumn{5}{|l|}{ Enucleation } \\
\hline Longest basal dimension (mm) & 0.047 & 1.190 & 1.002 & 1.412 \\
\hline Dose to foveola (Gy) & 0.000 & 1.010 & 1.005 & 1.015 \\
\hline \multicolumn{5}{|l|}{ Tumor shape } \\
\hline Mushroom (ref) & 0.001 & & & \\
\hline Nodular & 0.980 & 0.000 & 0.000 & \\
\hline Diffuse & 0.000 & 0.227 & 0.105 & 0.492 \\
\hline \multicolumn{5}{|l|}{ Location of anterior tumor border } \\
\hline Ciliary body (ref) & 0.011 & & & \\
\hline Equator to ora serrata & 0.008 & 0.222 & 0.073 & 0.678 \\
\hline Posterior to equator & 0.004 & 0.165 & 0.048 & 0.567 \\
\hline \multicolumn{5}{|l|}{ Disease-free survival } \\
\hline Longest basal dimension (mm) & 0.022 & 1.292 & 1.037 & 1.609 \\
\hline
\end{tabular}

84\% (95\% CI: 73-95\%), and 73\% (95\% CI: 63-84\%), respectively (Figure 1). Table 4 shows mortality rate.

The 3-, 5-, 10-, and 15-year melanoma-specific survival rates were 98\% (95\% CI: 96-100\%), 96\% (95\% CI: 93-99\%), 92\% (95\% CI: 84-96\%), and 87\% (95\% CI: 80-95\%), respectively (Figure 1). Eleven patients of the surviving cohort had acknowledged metastases at the end of follow-up (Table 4).

Univariate factors predictive of enucleation are listed in Table 5 showing that features associated with tumor and treatment, physical doses to de EC and BED to the lens were statistically significant. Multivariate logistic regression analysis, including age, revealed that only the longest basal dimension (HR: 1.292, 95\% CI: 1.037-1.609, $p=0.022$ ) was statistically significant (Table 6). BEDs dependencies vanished when multivariate competing risks regression modeling was performed.

\section{Discussion}

In this report, we present our experience after plaque brachytherapy and possible relationship with clinical features including radiobiological doses (among others) in a large series of patients from a single center. A homogeneous group of patients affected by uveal melanoma was selected. This study is the first to correlate radiobiological dose and local control, globe preservation, and survival by means of Dale's equation and Cox's proportional hazards models. A multicenter study involving larger patient population may confirm or disprove these findings.

A previous paper that assessed the long-term influence of radiobiological doses in the evolution of visual acuity (VA) in patients with the same cohort of patients was published recently by Miguel et al. [33].

\section{Local control}

In general, discovered survival rates will not be completely comparable to other studies, mainly due to differences in the selection of patients, tumor characteristics, staging, and different statistical approaches performed. A total of $94 \%$ of the patients in this study maintained actuarial local control at 5 years and is comparable - or higher - with values already reported by other authors: $89.7 \%$ by COMS report 18 [32] $(N=638), 92 \%(N=165)$ by Jensen et al. [34], 91\% by Pérez et al. [35] $(N=260)$, and $88.2 \%(N=120)$ by Correa et al. [36] for the same period of time. This confirms brachytherapy as a good treatment option for medium-sized melanomas.

In order to obtain an estimator of the effect size, we included age as forced variable in the Cox's proportional hazards models. Significant variables in the multivariate model for local control failure are the largest basal diameter, $\mathrm{HR}=1.23$ per millimeter and the juxtapapillary location, which triples the risk of local control failure. Interestingly, there is no direct association between local control and the physical and biological doses that are specifically administered to the tumor for destruction according to our protocol. This fact was also observed by others [37]. Some authors reported that lower doses $[35,38,39]$ should be sufficient to achieve acceptable local control rates in patients, and a radiobiological dose could play an important role when analyzing local control.

There can be many causes of local control failure. One of them is the proportion of tumor cells not sensitive to 
radiation. Either because of its genetic characteristics or its low mitotic index [40]. Another cause can be related to the margins of the tumor: The reference dose surrounds the tumor base with a $2 \mathrm{~mm}$ margin, so usually plaque sizes larger than the size at the longest basal diameter is chosen; however, the tumor growth patterns may be unequal. This can lead to a reduction in the coverage of the CTV considering the fact that the plaque can have a slight positioning inaccuracy [41]. We tried to minimize the impact by expanding the positioning safety margin of the applicator and verifying the position of the plaque by ultrasound image during the treatment. The association of increased risk of recurrence with close location to the optic disk may be related to challenges of plaque design and placement near the optic nerve.

In multivariate studies by different authors, significant values were obtained in many different features depending on the variables analyzed: tumor size $[32,37,42,43,44]$, older age [32,42], dose to the apex [32,43], the proximity of the tumor to the avascular zone of the fovea $[32,43]$, juxtapapillary location [45], use of TTT [42], tumor height [43], the distance from the margin of the tumor less than $2 \mathrm{~mm}$ from the optic disk [46], retinal invasion [46], and the location of the tumor margin within $3 \mathrm{~mm}$ to the macula [44].

\section{Eye preservation}

The preservation of the organ was maintained by majority of patients (95\%) at the end of the study. Five-year actuarial eye preservation was $89 \%$. The main reason for enucleation in the first 5 years was local recurrence; although after this period, most enucleations were associated with treatment-related complications, especially pain. The COMS report 18 [32] provides enucleation results of $12.5 \%$ at 5 years, $43 \%$ of them due to side effects, and $57 \%$ due to failure in local control. These results are very similar to ours. Some authors reported 5-year eye preservation rates of $72-82 \%[47,48]$.

For the analyzed event it was found that the largest diameter of the base, the mushroom form, the location in ciliary body, and the greater dose to the foveola were statistically significant unfavorable variables. The results of the present study show the association between enucleation and BED to tumor and critical tissues as statistically significant in univariate analysis but not in multivariate investigation.

Multivariate studies carried out by some authors obtained significant values in many different features depending on the variables analyzed: tumor size [44,32], distance to the fovea or macula [32], dose to apex [35], dose rates [49], age [32], and the distance to the fovea [32].

\section{Overall and disease-specific survival}

Because of non-censoring of non-melanoma deaths, it is possible to deduce from Kaplan-Meier curves patient's chances of dying from melanoma or chances of specified time surviving and separate then from the apparent disease-specific tumor mortality in order to avoid to a false impression of the survival probability. A remarkable fact is that the COMS report 18 [32] provides disease-specific mortality of $10 \%$ and $18 \%$ at 5 and 10 years, respectively.
This value is improved in our cohort because the survival rates were $95 \%$ and $90 \%$ for the same periods of time. The only risk factors for worse survival in multivariate analysis was the longest basal dimension. The results of the present study show the association between survival and BED to tumor and critical tissues as statistically significant but not in multivariate analysis. Multivariate studies carried out by some authors obtained as significant values in many different features depending on the variables analyzed: tumor size $[9,50,51]$ and ciliary body affectation [52].

\section{Study limitations}

Our retrospective study report on the treatment of patients with choroidal melanoma in Spain from 1996 to 2016. This study has several limitations. Only clinical factors have been considered but uveal melanoma prognosis has been shown to be dependent as well as histopathologic and cytogenetic factors and chromosomal mutations [53]. In contrast, some papers refer to beta-emitting plaques for comparison to ${ }^{125} \mathrm{I}$ results. While this comparison may be valid, the potential differences in dose distributions should be noted.

The main limitations of the radiobiological modeling are the lack of control of all the biological mechanisms involved in the expression of a certain radiation effect and the uncertainties in the formulation of the models from clinical data [15]. So, these models may not properly reflect normal tissue complications and as a result, the analysis could fail.

Secondly, Kaplan-Meier curves behave poorly in the tails, and the reliability of the estimates is intuitively bad when there are less than $10 \%$ of patients remaining in the cohort [54].

Cox models adjusted to use maximum possibility can perform poorly when certain events occur. Specific problems include predictions that are too extreme between low-risk and high-risk patients. The model can be unreliable if the datasets contain few events, which may be the case if either the disease or the event of interest is rare [55]. Despite these limitations, this review still provides valuable information regarding treatment factors that predict local control achievement, enucleation rates, and disease-specific survival after epiescleral brachytherapy.

\section{Conclusions}

Brachytherapy provides a good tumor control, a high globe preservation rates, and a relatively high 5-year survival. The reported results show that there is no clear radiobiological relation between radiobiological doses and local control, globe preservation and disease-specific survival in patients after brachytherapy.

By multivariate analysis, we concluded as significant variables: for local control failure: the longest basal diameter and the juxtapapillary location; for globe preservation failure: the largest basal dimension, the mushroom shape, the location in ciliary body, and the dose to the foveola; for disease-specific survival: the longest basal dimension. 


\section{Acknowledgements}

This study was partially supported by Varian Medical Systems Ibérica, S.L.

The authors would like to thank the referees for the valuable comments on our manuscript. D. Miguel's contributions as a part of his PhD thesis under the supervision of F. López-Lara and J. de Frutos-Baraja at the University of Valladolid.

\section{Disclosure}

The authors report no conflict of interest.

\section{References}

1. Virgili G, Gatta G, Ciccolallo L et al. Incidence of uveal melanoma in Europe. Ophthalmology 2007; 114: 2309-2315.

2. Shields JA, Shields CL, De Potter P. Approach to counseling patients with posterior uveal melanoma. Int Ophthalmol Clin 1993; 33: 143-145.

3. Gamel JW, McLean IW, McCurdy JB. Biologic distinctions between cure and time to death in 2892 patients with intraocular melanoma. Cancer 1993; 71: 2299-2305.

4. Kujala E, Mäkitie T, Kivelä T. Very long-term prognosis of patients with malignant uveal melanoma. Invest Ophthalmol Vis Sci 2003; 44: 4651-4659.

5. Diener-West M, Reynolds SM, Agugliaro DJ et al. Development of metastatic disease after enrollment in the COMS trials for treatment of choroidal melanoma: Collaborative Ocular Melanoma Study Group Report No. 26. Arch Ophthalmol 2005; 123: 1639-1643.

6. Rajpal S, Moore R, Karakousis CP. Survival in metastatic ocular melanoma. Cancer 1983; 52: 334-336.

7. Shields JA, Shields CL, Donoso LA. Management of posterior uveal melanoma. Surv Ophthalmol 1991; 36: 161-195.

8. Rao YJ, Sein J, Badiyan S et al. Patterns of care and survival outcomes after treatment for uveal melanoma in the post-coms era (2004-2013): a surveillance, epidemiology, and end results analysis. J Contemp Brachytherapy 2017; 9: 453-465.

9. Diener-West M, Earle JD, Fine SL et al. The COMS randomized trial of iodine 125 brachytherapy for choroidal melanoma, III: initial mortality findings. COMS Report No. 18. Arch Ophthalmol 2001; 119: 969-982.

10. Nag S, Quivey JM, Earle JD et al. The American Brachytherapy Society recommendations for brachytherapy of uveal melanomas. Int J Radiat Oncol Biol Phys 2003; 56: 544-555.

11. American Brachytherapy Society - Ophthalmic Oncology Task Force; Simpson ER, Gallie B, Laperrierre N et al. The American Brachytherapy Society consensus guidelines for plaque brachytherapy of uveal melanoma and retinoblastoma. Brachytherapy 2014; 13: 1-14.

12. Caswell RS, Deluca P, Seltzer SM et al. ICRU Report No. 72: Dosimetry of Beta Rays and Low-Energy Photons for Brachytherapy with Sealed Sources. Vol. 4.; 2004.

13. Krohn J, Monge OR, Skorpen TN et al. Posterior uveal melanoma treated with I-125 brachytherapy or primary enucleation. Eye (Lond) 2008; 22: 1398-1403.

14. Seregard S, Kock E. Prognostic indicators following enucleation for posterior uveal melanoma. A multivariate analysis of long-term survival with minimized loss to follow-up. Acta Ophthalmol Scand 1995; 73: 340-344.

15. Fowler JF. 21 years of Biologically Effective Dose. Br J Radiol 2010; 83: 554-568.

16. Fowler JF. The linear-quadratic formula and progress in fractionated radiotherapy. Br J Radiol 1989; 62: 679-694.
17. Dale RG. Some theoretical derivations relating to the tissue dosimetry of brachytherapy nuclides, with particular reference to iodine-125. Med Phys 1983; 10: 176-183.

18. Gagne NL, Leonard KL, Huber KE et al. BEDVH-A method for evaluating biologically effective dose volume histograms: application to eye plaque brachytherapy implants. Med Phys 2012; 39: 976-983.

19. Gagne NL, Leonard KL, Rivard MJ. Radiobiology for eye plaque brachytherapy and evaluation of implant duration and radionuclide choice using an objective function. Med Phys 2012; 39: 3332-3342.

20. Tagliaferri L, Pagliara MM, Boldrini L et al. INTERACTS (INTErventional Radiotherapy ACtive Teaching School) guidelines for quality assurance in choroidal melanoma interventional radiotherapy (brachytherapy) procedures. J Contemp Brachytherapy 2017; 9: 287-295.

21. Granero D, Pérez-Calatayud J, Ballester F et al. Dosimetric study of the $15 \mathrm{~mm}$ ROPES eye plaque. Med Phys 2004; 31: 3330-3336.

22. Chiu-Tsao ST, Astrahan MA, Finger PT et al. Dosimetry of (125)I and (103)Pd COMS eye plaques for intraocular tumors: report of Task Group 129 by the AAPM and ABS. Med Phys 2012; 39: 6161-6184.

23. Gagne NL, Rivard MJ. Quantifying the dosimetric influences of radiation coverage and brachytherapy implant placement uncertainty on eye plaque size selection. Brachytherapy 2013; 12: 508-520.

24. Astrahan MA, Luxton G, Jozsef $G$ et al. An interactive treatment planning system for ophthalmic plaque radiotherapy. Int J Radiat Oncol Biol Phys 1990; 18: 679-687.

25. Nath R, Anderson LL, Luxton G et al. Dosimetry of interstitial brachytherapy sources: recommendations of the AAPM Radiation Therapy Committee Task Group No. 43. American Association of Physicists in Medicine. Med Phys 1995; 22: 209-234.

26. Rivard MJ, Coursey BM, DeWerd LA et al. Update of AAPM Task Group No. 43 Report: A revised AAPM protocol for brachytherapy dose calculations. Med Phys 2004; 31: 633-674.

27. Dale RG, Jones B. The clinical radiobiology of brachytherapy. Br J Radiol 1998; 71: 465-483.

28. Verhoeff JJC, Stalpers LJA, Coumou AW et al. Experimental iodine- 125 seed irradiation of intracerebral brain tumors in nude mice. Radiat Oncol 2007; 2: 38.

29. Roberts SA, Hendry JH, Swindell R et al. Compensation for changes in dose-rate in radical low-dose-rate brachytherapy : a radiobiological analysis of a randomised clinical trial. Radiother Oncol 2004; 70: 63-74.

30. Bland JM, Altman DG. Survival probabilities (the KaplanMeier method). BMJ 1998; 317: 1572-1580.

31. Lunn M, McNeil D. Applying Cox regression to competing risks. Biometrics 1995; 51: 524-532.

32. Jampol LM, Moy CS, Murray TG et al. The COMS randomized trial of iodine 125 brachytherapy for choroidal melanoma: IV. Local treatment failure and enucleation in the first 5 years after brachytherapy. COMS report no. 19. Ophthalmology 2002; 109: 2197-2206.

33. Miguel D, de Frutos-Baraja JM, López-Lara F et al. Visual outcome after posterior uveal melanoma episcleral brachytherapy including radiobiological doses. J Contemp Brachytherapy 2018; 10: 123-131.

34. Jensen AW, Petersen IA, Kline RW et al. Radiation complications and tumor control after 125I plaque brachytherapy for ocular melanoma. Int J Radiat Oncol Biol Phys 2005; 63: 101-108.

35. Perez BA, Mettu P, Vajzovic L et al. Uveal melanoma treated with iodine-125 episcleral plaque: An analysis of dose on disease control and visual outcomes. Int J Radiat Oncol Biol Phys 2014; 89: 127-136. 
36. Correa R, Pera J, Gómez J et al. (125)I episcleral plaque brachytherapy in the treatment of choroidal melanoma: a single-institution experience in Spain. Brachytherapy 2009; 8: 290-296.

37. Damato B, Patel I, Campbell IR et al. Local tumor control after 106Ru brachytherapy of choroidal melanoma. Int J Radiat Oncol Biol Phys 2005; 63: 385-391.

38. Murray TG, Markoe AM, Gold AS et al. Long-term followup comparing two treatment dosing strategies of 125I plaque radiotherapy in the management of small/medium posterior uveal melanoma. J Ophthalmol 2013; 2013: 517032.

39. Gorovets D, Gagne NL, Melhus CS. Dosimetric and radiobiologic comparison of ${ }^{103} \mathrm{Pd}$ COMS plaque brachytherapy and Gamma Knife radiosurgery for choroidal melanoma. Brachytherapy 2017; 16: 433-443.

40. Kaiserman I, Anteby I, Chowers I et al. Changes in ultrasound findings in posterior uveal melanoma after Ruthenium 106 brachytherapy. Ophthalmology 2002; 109: 1137-1141.

41. Chang MY, McCannel TA. Local treatment failure after globeconserving therapy for choroidal melanoma. $\mathrm{Br} J$ Ophthalmol 2013; 97: 804-811.

42. Aziz HA, Singh N, Bena J et al. Vision Loss Following Episcleral Brachytherapy for Uveal Melanoma: Development of a Vision Prognostication Tool. JAMA Ophthalmol 2016; 134: 615-620.

43. Quivey JM, Char DH, Phillips TL et al. High intensity 125-iodine (125I) plaque treatment of uveal melanoma. Int J Radiat Oncol Biol Phys 1993; 26: 613-618.

44. Rospond-Kubiak I, Wróblewska-Zierhoffer M, TwardoszPawlik H. Ruthenium brachytherapy for uveal melanoma single institution experience. J Contemp Brachytherapy 2017; 9: 548-552.

45. Verschueren KMS, Creutzberg CL, Schalij-Delfos NE et al. Long-term outcomes of eye-conserving treatment with $\mathrm{Ru}-$ thenium106 brachytherapy for choroidal melanoma. Radiother Oncol 2010; 95: 332-338.

46. Gündüz K, Shields CL, Shields JA et al. Radiation complications and tumor control after plaque radiotherapy of choroidal melanoma with macular involvement. Am J Ophthalmol 1999; 127: 579-589.

47. Bergman L, Nilsson B, Lundell G et al. Ruthenium Brachytherapy for Uveal Melanoma, 1979-2003 Survival and Functional Outcomes in the Swedish Population. Ophthalmology 2005; 112: 834-840.

48. Isager P, Ehlers N, Urbak SF et al. Visual outcome, local tumour control, and eye preservation after ${ }^{106} \mathrm{Ru} / \mathrm{Rh}$ brachytherapy for choroidal melanoma. Acta Oncol 2009; 45: 285-293.

49. Fili M, Lundell G, Lundell $M$ et al. High dose rate and low dose rate ruthenium brachytherapy for uveal melanoma. No association with ocular outcome. Br J Ophthalmol 2014; 98: 1349-1354.

50. Petrovich Z, Luxton G, Langholz B et al. Episcleral plaque radiotherapy in the treatment of uveal melanomas. Int J Radiat Oncol Biol Phys 1992; 24: 247-251.

51. Shields CL, Kaliki S, Cohen MN et al. Prognosis of uveal melanoma based on race in 8100 patients: The 2015 Doyne Lecture. Eye (Lond) 2015; 29: 1027-1035.

52. McLean MJ, Foster WD, Zimmerman LE. Prognostic factors in small malignant melanomas of choroid and ciliary body. Arch Ophthalmol 1977; 95: 48-58.

53. Kaliki S, Shields CL, Shields JA. Uveal melanoma: estimating prognosis. Indian J Ophthalmol 2015; 63: 93-102.

54. Rich JT, Neely JG, Paniello RC et al. A practical guide to understanding Kaplan-Meier curves. Otolaryngol Head Neck Surg 2010; 143: 331-336.

55. Ambler G, Seaman S, Omar RZ. An evaluation of penalised survival methods for developing prognostic models with rare events. Stat Med 2012; 31: 1150-1161. 